\title{
Uso de Grupos Focales como Complemento del Método CoPso0 PSOCAT de Evaluación de Factores de Riesgos Psicosociales
}

\author{
USE OF FOCAL GROUPS AS A COMPLEMENT OF THE COPSOQ PSOCAT METHOD'S OF EVALUATION OF \\ PSYCHOSOCIAL RISK FACTORS
}

Pablo César Gutiérrez Falcón

Universidad Tecnológica del Perú. Lima - Perú

\section{RESUMEN}

El método CoPsoQ PSQCAT Versión Corta permite conocer la percepción de los trabajadores sobre las dimensiones de riesgos psicosociales, a través del uso de un cuestionario estandarizado; sin embargo, es necesario triangular las fuentes de información para determinar los factores de riesgo psicosocial que afectan a los trabajadores de una organización.

La investigación descriptiva-transversal tuvo por objetivo examinar y ampliar los resultados obtenidos provenientes del método CoPsoQ PSQCAT Versión Corta, complementándolo con la técnica de grupos focales.

Los resultados obtenidos al introducir un grupo focal, después de aplicar el proceso de intervención del método CoPsoQ PSQCAT Versión Corta en una empresa de menos de 25 trabajadores, fueron la validación de la clasificación de 11 dimensiones de riesgo psicosocial, la clasificación de 2 dimensiones con resultados no concluyentes, y la modificación de la clasificación de otras 2 dimensiones. Se concluyó que la aplicación de un grupo focal permitió profundizar en el análisis de la información obtenida a partir del cuestionario del método CoPsoQ PSQCAT Versión Corta para la identificación y evaluación de los factores de riesgo psicosocial.

(Gutiérrez P, 2017. Uso de Grupos Focales como Complemento del Método CoPsoQ PSQCAT de Evaluación de Factores de Riesgos Psicosociales. Cienc Trab. Sep-Dic; 19 [60]: 166-170).

Palabras Clave: SALUD LABORAL, CONDICIONES DE TRABAJO, FACTORES DE RIESGO, SATISFACCIÓN EN EL TRABAJO, PSICOLOGÍA INDUSTRIAL.

\section{ABSTRACT}

The CoPsoQ PSQCAT method's (Short Version) allows knowing the perception of workers about the dimensions of psychosocial risks, through the use of a standardized questionnaire; however, it is necessary to triangulate the sources of information to determine the psychosocial risk factors that affect the workers of an organization. The objective of the descriptive - transversal research was to examine and extend the results obtained from the CoPsoQ PSQCAT method's (Short Version), complementing it with focus group.

The results obtained by introducing a focus group, after applying the process of intervention of the CoPsoQ PSQCAT method's in a company with fewer than 25 workers, method allowed to validate the classification of 11 dimensions of psychosocial risk, in addition to classify 2-dimensional with inconclusive results and modify rated 2 other dimensions.

It was concluded that the application of a focus group allowed to deepen the analysis of the information obtained from the questionnaire of the CoPsoQ PSQCAT method's (Short Version) for the identification and evaluation of psychosocial risk factors,

Key Words: OCCUPATIONAL HEALTH, WORKING CONDITIONS, RISK FACTORS, JOB SATISFACTION, PSYCHOLOGY INDUSTRIAL.

\section{INTRODUCCIÓN}

Raffo et $\mathrm{al}^{1}$ concluyeron que la legislación peruana ha avanzado en la materia de Prevención de Riesgos Laborales (PRL) y señalaron que la Ley N² 29783 -Ley de Seguridad y Salud en el Trabajo- promueve una cultura de la PRL. Entre las buenas intenciones de la Ley N 29783 está la obligación de los empleadores públicos o privados respecto a la prevención de daños en la salud de los trabajadores por exposición

\section{Correspondencia / Correspondence:}

Pablo César Gutiérrez Falcón

Av. Belisario Suárez 948 San Juan de Miraflores, Lima - Perú

Código Postal: 15801

Tel.: (51-1) 997369253

e-mails: pcgutierrezf@gmail.com•pgutierrezf@copan-sgi.com

Recibido: 02 de Agosto de 2017 / 05 de Noviembre de 2017

a agentes físicos, químicos, biológicos, disergonómicos y psicosociales en el centro de trabajo. LaLey N 29783 está complementada por normas y guías en varias áreas (Tabla 1), pero carece de guías metodológicas sobre cómo actuar en la identificación y evaluación de los riesgos psicosociales.

La Dirección General de Relaciones Laborales de Cataluña ${ }^{2}$ denomina factores de riesgo psicosocial a aquellos factores de riesgo para la salud que se originan en la organización del trabajo y que generan lo que conocemos como estrés*, y que puede ser precursor de enfermedades en ciertas circunstancias de intensidad, frecuencia y duración. Los factores de riesgo psicosociales representan la exposición, la organización del trabajo representa el origen de esta exposición, y el estrés es el precursor o antecesor del efecto (la enfermedad o trastorno de salud) que se ha de evitar.

* Mc Grath (1970): El estrés es un desequilibrio sustancial (percibido) entre la demanda y la capacidad de respuesta (del individuo) bajo condiciones en la que el fracaso ante esta demanda posee importantes consecuencias (percibidas)". 
Tabla 1.

Normativa peruana básica relacionada a los Agentes y Factores de Riesgos.

\begin{tabular}{|c|c|}
\hline $\begin{array}{l}\text { Agente / } \\
\text { Factor de } \\
\text { Riesgo }\end{array}$ & Normativa Relacionada \\
\hline Físico & $\begin{array}{l}\text { - Norma Básica de Ergonomía y del Procedimiento de Evaluación del } \\
\text { Riesgo Disergonómico (Resolución Ministerial № 375-2008-TR). } \\
\text { - Reglamento de Seguridad y Salud Ocupacional en Minería (Decreto } \\
\text { Supremo Nº24-2016-EM). }\end{array}$ \\
\hline Químico & $\begin{array}{l}\text { - Reglamento sobre Valores Limite Permisibles para Agentes Quimicos en } \\
\text { el Ambiente de Trabajo (Decreto Supremo No 015-2005-SA). } \\
\text { - Reglamento de Seguridad y Salud Ocupacional en Mineria (Decreto } \\
\text { Supremo N024-2016-EM). }\end{array}$ \\
\hline Biológico & $\begin{array}{l}\text { - Guía Técnica para el Análisis Microbiológico de Superficies en contacto } \\
\text { con Alimentos y Bebidas (Resolución Ministerial N 461-2007-MINSA). } \\
\text { - Norma Sanitaria que establece los criterios microbiológicos de calidad } \\
\text { sanitaria e inocuidad para los alimentos y bebidas de consumo humano } \\
\text { (Resolución Ministerial } N^{\circ} \text { 591-2008-MINSA). }\end{array}$ \\
\hline Ergonómico & $\begin{array}{l}\text { - Norma Básica de Ergonomía y del Procedimiento Básico de Evaluación } \\
\text { del Riesgo Disergonómico (Resolución Ministerial № 375-2008-TR). }\end{array}$ \\
\hline
\end{tabular}

Además, la Dirección General de Relaciones Laborales de Cataluña ${ }^{2}$ precisa que la exposición laboral a factores psicosociales es una de las causas más relevantes de ausentismo laboral por motivos de salud, y se ha relacionado muy especialmente con las enfermedades cardiovasculares, y trastornos de salud mental y músculo-esqueléticos. También se le ha relacionado con otros trastornos y conductas poco saludables como el hábito de fumar, el consumo de alcohol y drogas y el sedentarismo.

El Instituto Nacional de Seguridad e Higiene en el Trabajo ${ }^{3}$ y la Dirección General de Relaciones Laborales de Cataluña ${ }^{2}$ han hecho un esfuerzo por atender a las pequeñas y medianas empresas desarrollando instrumentos de aplicación práctica. Sin embargo, no muestran cómo gestionar los riesgos psicosociales. Bravo y Eggerstedt ${ }^{4}$ ofrecen los resultados de una encuesta que realizaron sobre riesgos psicosociales en 130 trabajadores de diversas profesiones y ocupaciones de Lima; ellos reportaron la prevalencia de estrés laboral de la población estudiada (30,5\%), así como del síndrome de burnout $(14,4 \%)^{* *}$.

López M. et $\mathrm{al}^{5}$ encontraron que el 10,75\% de la población de un hospital de Lima presentaba una alta exposición a riesgos psicosociales y el 62,92\% una exposición media, mientras que el resto $(26,33 \%)$ se encuentra en condiciones adecuadas. La mala remuneración del rendimiento $(31,2 \%)$ y las exigencias laborales $(15,9 \%)$ fueron los mayores factores de riesgo psicosocial.

El Departamento de Empresa y Empleo de la Generalitat de Catalunya (DEMO) efectuó la adaptación de la metodología internacional COPSOQ, desarrollada originariamente por el National Research Centre for the Working Environment de Dinamarca, poniendo a disposición pública el método CoPsoQ PSQCAT para la evaluación de los riesgos psicosociales en castellano.

El método CoPsoQ PSQCAT dispone de dos versiones: la versión media para empresas con 25 personas o más y la versión corta para empresas con menos de 25 personas. La versión corta identifica y valora 15 dimensiones de riesgos psicosociales: Exigencias psicoló-

\footnotetext{
** También llamado "Síndrome de estar quemado por el trabajo" es una respuesta al estrés laboral crónico integrada por actitudes y sentimientos negativos hacia las personas con las que se trabaja y hacia el propio rol profesional, así como por la vivencia de encontrarse emocionalmente agotado. Esta respuesta ocurre con frecuencia en los profesionales de la salud y, en general, en profesionales de organizaciones de servicios que trabajan en contacto directo con los usuarios de la organización.
}

gicas cuantitativas, Ritmo de trabajo, Exigencias psicológicas emocionales, Doble presencia, Influencia, Posibilidad de desarrollo, Sentido del trabajo, Calidad de liderazgo, Previsibilidad, Claridad de rol, Conflicto de rol, Inseguridad sobre el empleo, Inseguridad sobre las condiciones de trabajo, Confianza vertical, y Justicia. Esta versión, gestionada por el DEMO, está acompañada por un proceso de intervención explícito y específico (Tabla 2). En la etapa 2 "Obtener los datos de exposición” del proceso de intervención del método CoPsoQ PSQCAT se establece, entre otras actividades, que se debe informar a los trabajadores sobre los riesgos psicosociales y el proceso de intervención preventiva. Para ello el método propone que se utilice mecanismos por escrito, además de una charla presencial antes de la aplicación del cuestionario.

\section{Tabla 2.}

Proceso de Intervención del Método CoPsoO PSOCAT.

\begin{tabular}{|c|c|}
\hline Etapa & ¿Qué se Hace? \\
\hline $\begin{array}{l}\text { Acordar la evaluación } \\
\text { y prevención de los } \\
\text { riesgos psicosociales. }\end{array}$ & $\begin{array}{l}\text { - Aceptar la licencia de uso del método: implica el acuerdo de } \\
\text { hacer la evaluación de riesgos psicosociales con el CoPso0 } \\
\text { PSOCAT, versión corta, respetando las condiciones } \\
\text { alli establecidas. } \\
\text { - De este acuerdo se debe informar a los trabajadores. }\end{array}$ \\
\hline $\begin{array}{l}\text { Obtener los datos } \\
\text { de exposición. }\end{array}$ & $\begin{array}{l}\text { - Diseñar la distribución, respuesta y recogida del cuestionario. } \\
\text { - Informar a los trabajadores. } \\
\text { - Distribuir y recoger el cuestionario. } \\
\text { - Resumir los datos de exposición. }\end{array}$ \\
\hline $\begin{array}{l}\text { Acordar y llevar a } \\
\text { cabo las medidas } \\
\text { preventivas. }\end{array}$ & $\begin{array}{l}\text { - Concretar el origen de la exposición y una propuesta de } \\
\text { medidas preventivas. } \\
\text { - Acordar, planificar y realizar segui-miento de las } \\
\text { medidas preventivas. }\end{array}$ \\
\hline
\end{tabular}

Fuente: Adaptación de Proceso de Intervención del Método CoPsoQ PSOCAT publicado en Manual del Método CoPsoQ PSOCAT (versión 2). p.15-16).

Schaufeli y Salanova ${ }^{3}$ señalaron que los cuestionarios son subjetivos por naturaleza, y ello significa que la evaluación y valoración de riesgos está basada en las experiencias de estrés de los trabajadores y no en las características del puesto de trabajo en sí mismo. Mediante la agregación de puntuaciones de los trabajadores individuales a nivel del puesto o de la unidad de trabajo, y por comparación con otras unidades de trabajo o puestos similares, pueden evaluarse los riesgos psicosociales relativos a un determinado puesto de trabajo o unidad. Sin embargo, en la licencia de uso del método CoPsoQ PSQCAT se establece que el cuestionario no se puede modificar, es decir, no es posible variar la formulación de las preguntas, ni añadir o eliminar ninguna pregunta, además de que se debe garantizar el anonimato y la confidencialidad de los datos individuales. Así, pues, existe la posibilidad que tras la aplicación del cuestionario se pueda obtener resultados no concluyentes sobre dimensiones de riesgos psicosociales, en el sentido que tras la contabilización de las respuestas a las preguntas de opción múltiple no sea posible determinar si estas dimensiones representan una situación más favorable para la salud, una situación intermedia o una situación más desfavorable para la salud.

Charria et $\mathrm{al}^{6}$ señalaron que diversos autores proponen la triangulación de las fuentes de información para la evaluación de los riesgos psicosociales: los resultados estadísticos de una escala orientada a identificar la percepción de los sujetos, los registros objetivos de evaluación en el puesto de trabajo y las metodologías cualitativas complementarias como entrevistas, grupos focales y observación participante.

Gibb señala que el propósito principal de los grupos focales*** es hacer que surjan actitudes, sentimientos, creencias, experiencias y reacciones en los participantes, lo cual no es fácil de lograr con otros 
métodos. Gibbs agrega que los grupos focales, comparados con la entrevista individual, permiten obtener una multiplicidad de miradas y procesos emocionales dentro del contexto del grupo. Frente a lo señalado, se planteó la siguiente pregunta: ¿la técnica de grupos focales aportará información que sirva de complemento al CoPsoQ PSQCAT?

El presente estudio se planteó el objetivo de examinar y ampliar los resultados de la identificación y evaluación de los factores de riesgos psicosociales obtenidos, a través del método CoPsoQ PSQCAT, complementado por grupos focales. El estudio representa un aporte para las empresas con menos de 25 trabajadores dado que brinda un modelo para la identificación y evaluación de los factores de riesgo psicosocial y el control de los mismos.

\section{MATERIAL Y MÉTODOS}

\section{Empresa de Estudio}

La investigación tomó como caso de aplicación a una empresa familiar dedicada a la formación, capacitación, evaluación y certificación de operadores de maquinaria pesada, flota auxiliar y equipos de izaje; así como en la inspección, evaluación y certificación de operatividad de maquinaria pesada, flota auxiliar y equipos de izaje; para los sectores de minería, construcción, agroindustria u otros rubros.

La planilla de la empresa de estudio consta de 12 trabajadores (02 gerentes y 10 trabajadores sin personal a cargo). Participaron en el estudio 7 trabajadores (sin incluir gerentes) debido a que 3 trabajadores se encontraban en comisión de servicios fuera de la ciudad de Lima. Esto representa una tasa de respuesta del 70\%.

\section{Proceso de Intervención - Método CoPsoQ PSQCAT Versión Corta}

Para la identificación y evaluación de los factores de riesgo psicosociales se aplicó el proceso de intervención propuesto por el método CoPsoQ PSQCAT Versión Corta (Tabla 2).

\section{Introducción del Grupo Focal}

En la etapa "Obtención de los datos de la exposición" del proceso de intervención por el método CoPsoQ PSQCAT Versión Corta, se procedió bajo la siguiente pauta:

- Se brinda información a los trabajadores sobre los riesgos psicosociales y el proceso de evaluación.

- Aplicación del cuestionario estandarizado (versión corta) que consta de 30 preguntas correspondientes a 15 dimensiones de exposición a riesgos psicosociales (anónimo).

- Consolidar los datos del cuestionario estandarizado.

Una vez consolidado los resultados del cuestionario estandarizado, se introdujo el uso de un grupo focal bajo la dirección de un profesional de psicología organizacional para profundizar en el análisis tomando en cuenta los siguientes aspectos:

- Población: Trabajadores de la empresa de estudio.

- Muestra: $100 \%$ de los trabajadores que cumplan los siguientes criterios de selección

- Trabajadores con relación laboral vigente.

\footnotetext{
****Esta modalidad de entrevista grupal es abierta y estructurada: generalmente toma la forma de una conversación grupal, en la cual el investigador plantea algunas temáticas-preguntas asociadas a algunos antecedentes que orientan la dirección de la misma, de acuerdo con los propósitos de la investigación (Aigneren, 2002). ${ }^{7}$
}

- Trabajadores a tiempo completo.

- Trabajadores sin personal a cargo.

- Tiempo de servicios no menor a 3 meses (trabajadores que hayan superado el periodo de prueba según lo dispuesto en la legislación laboral).

- Grupos focales: Se conforman según el tiempo de servicios (menos de 1 año, más de 1 año y más de 2 años).

- Técnica de análisis: Análisis de contenido.

\section{RESULTADOS}

\section{Resultados de la Aplicación del Cuestionario Estandarizado}

Los factores de riesgo psicosocial que representan una "Situación más desfavorable" para la salud de los trabajadores fueron: Exigencias cuantitativas, Ritmo de trabajo, Conflicto de rol, Previsibilidad, Inseguridad sobre el trabajo, y Justicia.

Los factores de riesgo psicosociales que representan una "Situación intermedia" para la salud de los trabajadores: Influencia, Inseguridad sobre las condiciones de trabajo, y Confianza vertical.

Los factores de riesgo psicosociales que representan una "Situación más favorable" para la salud de los trabajadores: Doble presencia, Exigencias emocionales, Posibilidades de desarrollo, y Sentido del trabajo.

En cuanto a los factores "Claridad del rol" y "Calidad de Liderazgo", los resultados del cuestionario estandarizado no fueron concluyentes, dado que no es posible determinar si estos corresponden a una "Situación intermedia" o "Situación más desfavorable para la salud" porque ninguna de estas posibles situaciones comprende más del 50\% de las opiniones de los trabajadores.

Grupo Focal: Examen y Ampliación de los Resultados del Cuestionario Estandarizado.

Tras la realización del grupo focal, se obtuvo los siguientes resultados:

- Validar la situación de exposición de 11 dimensiones de riesgo psicosocial.

Tabla 3.

Resultados cuantitativos según cantidad de cuestionarios por dimensión.

\begin{tabular}{|c|c|c|c|}
\hline Dimensiones & $\begin{array}{l}\text { ro de cuest } \\
\text { lación más } \\
\text { rable para } \\
\text { a salud }\end{array}$ & en cada sitı & $\begin{array}{l}\text { n de exposición } \\
\text { Situación más } \\
\text { desfavorable } \\
\text { para la salud }\end{array}$ \\
\hline 1 Exigencias cuantitativas & 1 & 2 & 4 \\
\hline 2 Doble presencia & 6 & 1 & 0 \\
\hline 3 Exigencias emocionales & 6 & 1 & 0 \\
\hline 4 Ritmo de trabajo & 0 & 0 & 7 \\
\hline 5 Influencia & 3 & 4 & 0 \\
\hline 6 Posibilidades de desarrollo & 5 & 2 & 0 \\
\hline 7 Sentido del trabajo & 6 & 0 & 1 \\
\hline 8 Claridad de rol & 2 & 3 & 2 \\
\hline 9 Conflicto de rol & 0 & 3 & 4 \\
\hline 10 Previsibilidad & 0 & 1 & 6 \\
\hline $\begin{array}{l}11 \text { Inseguridad sobre } \\
\text { las condiciones de trabajo }\end{array}$ & 1 & 6 & 0 \\
\hline 12 Inseguridad sobre el trabajo & 1 & 1 & 5 \\
\hline 13 Confianza vertical & 1 & 5 & 1 \\
\hline 14 Justicia & 1 & 2 & 4 \\
\hline 15 Calidad del liderazgo & 1 & 3 & 3 \\
\hline
\end{tabular}

Elaboración propia. 
Tabla 4.

Resultados del Cuestionario Estandarizado y Grupo Focal.

\begin{tabular}{|c|c|c|c|}
\hline \multirow{2}{*}{ Dimensiones } & \multicolumn{2}{|c|}{ Resultados } & \multirow{2}{*}{ Estado } \\
\hline & Cuestionario & Grupo Focal & \\
\hline Exigencias cuantitativas & Más Desfavorable & Más Desfavorable & Validado \\
\hline Doble presencia & Más Favorable & Más Favorable & Validado \\
\hline Exigencias emocionales & Más Favorable & Más Favorable & Validado \\
\hline Ritmo de trabajo & Más Favorable & Más Favorable & Validado \\
\hline Influencia & Intermedia & Intermedia & Validado \\
\hline Posibilidades de desarrollo & Más Favorable & Más Favorable & Validado \\
\hline Sentido del trabajo & Más Favorable & Más Favorable & Validado \\
\hline Claridad de rol & Sin Clasificar & Más Desfavorable & Clasificado \\
\hline Conflicto de rol & Más Desfavorable & Más Desfavorable & Validado \\
\hline Previsibilidad & Más Desfavorable & Más Desfavorable & Validado \\
\hline $\begin{array}{l}\text { Inseguridad sobre las } \\
\text { condiciones de trabajo }\end{array}$ & Intermedia & Intermedia & Validado \\
\hline el trabajo & Más Desfavorable & Más Favorable & Modificado \\
\hline Confianza vertical & Intermedia & Intermedia & Validado \\
\hline Justicia & Más Desfavorable & Intermedia & Modificado \\
\hline Calidad del liderazgo & Sin Clasificar & Más Desfavorable & Clasificado \\
\hline
\end{tabular}

Elaboración propia.

- Determinar la situación de exposición de 02 dimensiones de riesgo psicosocial cuyos resultados no fueron concluyentes (Claridad de rol y Calidad de liderazgo).

- Modificar la situación de exposición de 02 dimensiones de riesgo psicosocial (Inseguridad en el trabajo y Justicia).

\section{DISCUSIÓN DE RESULTADOS}

A través del método CoPsoQ PSQCAT Versión Corta se determinó la percepción de los trabajadores sobre 13 dimensiones de riesgos psicosociales; sin embargo, esto no fue posible para 2 dimensiones debido a que sus resultados no fueron concluyentes. Además, no fue posible obtener resultados objetivos de la evaluación en los puestos de trabajo en virtud de las disposiciones de su licencia de uso (confidencialidad y anonimato).

En pro de la triangulación de las fuentes de información propuesta por Charria, Sarsosa y Arenas ${ }^{6}$, se aplicó un Grupo Focal como una metodología cualitativa complementaria. Con esta metodología, se logró clasificar la situación de exposición de las 2 dimensiones de riesgo psicosocial con resultados no concluyentes y, además, se modificó la clasificación de otras 2 dimensiones. Esto fue posible dado que, a través del grupo focal, se recogió y examinó información con mayor detalle sobre las dimensiones de riesgo psicosocial proporcionada por los mismos trabajadores que participaron en el proceso de intervención del método CoPsoQ PSQCAT:

- La dimensión Claridad de rol se clasificó como una situación más desfavorable para la salud de los trabajadores porque se identificó que en la empresa no estaban claramente definidas las funciones de los trabajadores. Esta situación originaba que los trabajadores desempeñen actividades que no eran de su responsabilidad.
- La dimensión Inseguridad sobre el trabajo se modificó de una situación más desfavorable para la salud a una situación más favorable debido a que los trabajadores manifestaron que no se sienten vulnerables frente a despidos arbitrarios porque consideran que si realizan bien su trabajo, no tienen que temer.

- La dimensión Justicia se modificó de una situación más desfavorable para la salud a una situación intermedia debido a que, según manifestaron los trabadores, se evidencia diferencias en trato hacia los trabajadores, ya sea en la comunicación y en la paciencia frente a posibles errores.

- La dimensión Calidad de liderazgo se clasificó como una situación más desfavorable para la salud de los trabajadores por la poca capacidad de planificación y organización de la gerencia, y de tolerar los puntos de vista diferentes.

Concluido esta evaluación, se inició la etapa "Acordar y llevar a cabo las medidas preventivas" del proceso de intervención que se concretó en recomendaciones para abordar las dimensiones que representaban una situación más desfavorable para la salud.

Con esta investigación, se concluye que:

- A través de la aplicación del método CoPsoQ PSQCAT Versión Corta se obtiene resultados preliminares sobre las dimensiones de riesgo psicosociales.

- La aplicación de un grupo focal permite profundizar en el análisis de la información preliminar sobre las dimensiones de riesgo psicosociales contribuyendo al proceso de identificación y evaluación de los factores de riesgo psicosocial en empresas con menos de 25 trabajadores.

Esta investigación pueda dar paso al desarrollo de futuros estudios para la adecuación del método CoPsoQ PSQCAT Versión Corta para la identificación y evaluación de los factores de riesgo psicosocial en el Perú, dado que actualmente no se cuenta con una metodología validada por las autoridades competentes; a pesar de que la legislación peruana requiere del monitoreo de los factores de riesgos psicosocial.

La principal limitación para esta investigación ha sido la dificultad de introducir los grupos focales en los procesos de intervención en otras empresas, debido a dos factores:

- Desconocimiento de los empresarios de la obligación legal de efectuar monitoreos de factores de riesgo psicosocial, y

- El temor de las gerencias por los resultados de este tipo de estudios.

\section{Agradecimientos}

A la Licenciada en Psicología Consuelo Vicario, quien dirigió el grupo focal y contribuyó con recomendaciones para la implementación de medidas preventivas para abordar los factores de riesgos psicosociales.

Tema presentado en el VI Congreso Peruano de Salud Ocupacional Piura - Perú, 23/11/2016

Fuente de Financiamiento: Consorcio Ópalo Andino E.I.R.L.(COPAN). 


\section{REFERENCIAS}

1. Raffo E, Ráez L, Cachay O. Riesgos Psicosociales. Ind data. 2013;16(1):70-79.

2. Gobierno de Cataluña. Manual del método CoPsoQ PSQCAT (versión 2) para la evaluación y prevención de los riesgos psicosociales en las empresas de menos de 25 trabajadores y trabajadoras (versión corta). Barcelona: Gobierno de Cataluña; 2015.

3. Schaufeli W, Salanova M. ¿Cómo evaluar los riesgos psicosociales en el trabajo? Preven Trab Salud. 2002; (20):4-10.

4. Bravo E, Eggerstedt M. Los riesgos psicosociales en los ambientes laborales peruanos. Infocapital humano.pe [Pag Web]. Lima EDB Group; 2014 [citado sep 2017]. Disponible en: http://www.infocapitalhumano.pe/recursos-humanos/ articulos/los-riesgos-psicosociales-en-los-ambientes-laborales-peruanos/\#

5. López MR, García S, Pando M. Factores de riesgo psicosocial y burnout en población económicamente activa de Lima, Perú. Cien Trab. 2014;16(51):164-169.

6. Charria V, Sarsosa K, Arenas, F. Factores de riesgo psicosocial laboral: métodos e instrumentos de evaluación. Rev Fac Nac Salud Pública. 2011; 29(4):380-391.

7. Aigneren M. La Técnica de recolección de información mediante Grupos Focales. Sociol Escena [en línea]. 2002 [citado oct 2016]; 5(6):1-32. Disponible en: http://aprendeenlinea.udea.edu.co/revistas/index.php/ceo/ article/viewArticle/1611
Referencias complementarias:

Cook T., Reichardt C. Métodos cualitativos y cuantitativos en investigación evaluativa. Madrid: Morata; 1986.

Escobar J, Bonilla-Jiménez F. Grupos focales: una guia conceptual y metodológica. Cuad hispanoamer psicol. 2009. 9(1):51-67.

Estreslaboral.INFO [sitio Web]. ¿Qué es el estrés laboral? [d.d.] [citado 3 oct 2016].Disponible en: http://www.estreslaboral.info

Fidalgo M. NTP 704. Sindrome de estar quemado por el trabajo o "burnout" (I): definición y proceso de generación. Barcelona: INSHT; 200?

Kawulich B. La observación participante como método de recolección de datos. Forum Qual Soc Res [en línea] 2006 [citado 3 oct 2016]; 6(1), art.43. Disponible en: ttp://diverrisa.es/uploads/documentos/LA-OBSERVACIONPARTICIPANTE.pdf

Martín F. NTP 318. El estrés: proceso de generación en el ámbito laboral. Barcelona: INSHT;199?

Reyes T. Métodos Cualitativos de Investigación: Los Grupos Focales y el Estudio de Casos [en línea].[d.d.] [citado 3 oct 2016]. Disponible en: <http://jbposgrado.org/icuali/Estudio\%20de\%20caso\%20y\%20grupo\%20focal.pdf> 